\title{
BMJ Open Sequential simulation (SqS) of clinical pathways: a tool for public and patient engagement in point-of-care diagnostics
}

\author{
Jeremy R Huddy, ${ }^{1}$ Sharon-Marie Weldon, ${ }^{1}$ Shvaita Ralhan, ${ }^{1}$ Tim Painter, ${ }^{2}$ \\ George B Hanna, ${ }^{1}$ Roger Kneebone, ${ }^{1}$ Fernando Bello ${ }^{1}$
}

To cite: Huddy JR,

Weldon S-M, Ralhan S, et al. Sequential simulation (SqS) of clinical pathways: a tool for public and patient engagement in point-of-care diagnostics. BMJ Open 2016;6:e011043.

doi:10.1136/bmjopen-2016011043

- Prepublication history and additional material is available. To view please visit the journal (http://dx.doi.org/ 10.1136/bmjopen-2016011043).

Received 5 January 2016 Revised 3 May 2016 Accepted 20 July 2016

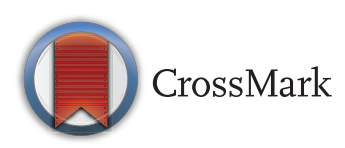

${ }^{1}$ Department of Surgery and Cancer, Imperial College, London, UK

${ }^{2}$ Patient Representative, London Cancer Alliance, London, UK

Correspondence to Jeremy R Huddy; j.huddy@imperial.ac.uk

\section{ABSTRACT}

Objectives: Public and patient engagement (PPE) is fundamental to healthcare research. To facilitate effective engagement in novel point-of-care tests (POCTs), the test and downstream consequences of the result need to be considered. Sequential simulation $(\mathrm{SqS})$ is a tool to represent patient journeys and the effects of intervention at each and subsequent stages. This case study presents a process evaluation of SqS as a tool for PPE in the development of a volatile organic compound-based breath test POCT for the diagnosis of oesophagogastric $(\mathrm{OG})$ cancer.

Setting: Three 3-hour workshops in central London. Participants: 38 members of public attended a workshop, $26(68 \%)$ had no prior experience of the $O G$ cancer diagnostic pathway.

Interventions: Clinical pathway SqS was developed from a storyboard of a patient, played by an actor, noticing symptoms of oesophageal cancer and following a typical diagnostic pathway. The proposed breath testing strategy was then introduced and incorporated into a second SqS to demonstrate pathway impact. Facilitated group discussions followed each SqS.

Primary and secondary outcome measures: Evaluation was conducted through pre-event and postevent questionnaires, field notes and analysis of audiovisual recordings.

Results: 38 participants attended a workshop. All participants agreed they were able to contribute to discussions and like the idea of an $O G$ cancer breath test. Five themes emerged related to the proposed new breath test including awareness of $O G$ cancer, barriers to testing and diagnosis, design of new test device, new clinical pathway and placement of test device. 3 themes emerged related to the use of SqS: participatory engagement, simulation and empathetic engagement, and why participants attended.

Conclusions: SqS facilitated a shared immersive experience for participants and researchers that led to the coconstruction of knowledge that will guide future research activities and be of value to stakeholders concerned with the invention and adoption of POCT.

\section{BACKGROUND}

Public and patient engagement (PPE) is a fundamental component of healthcare

\section{Strengths and limitations of this study}

- This article presents the use of sequential simulation (SqS) as a tool for public and patient engagement in new point-of-care diagnostic testing strategies.

- SqS allows participants to appreciate the patient journey, including the possible downstream pathway consequences of introducing new diagnostic strategies.

- This shared immersion of patients, publics and researchers allows coconstruction of knowledge that will guide future research, evidence generation and policy.

- Patient engagement necessitates voluntary participation and convenience sampling was unavoidable.

- The test device under evaluation is at a relatively early stage of development and therefore the $\mathrm{SqS}$ scenarios were based on assumptions.

research and actively encouraged by the Department of Health and major funding bodies. PPE refers to the dissemination of information and knowledge between healthcare providers and researchers, members of the public (including patients, carers and people who use health and social care services) and members of organisations representing service users. ${ }^{1}$ This participation ensures research is applicable to patients, and therefore, increases the likelihood that research findings will translate into clinical practice $^{2}$ as well as addressing the ethical and political requirements of PPE within healthcare research. ${ }^{3}{ }^{4}$ Involving patients in the management of their healthcare is widely recognised to improve quality of care, patient safety and health outcomes. ${ }^{5-8}$ However, PPE can be expensive and, if not rigorously undertaken with appropriate methodologies, there is a risk of reducing it to a mechanistic 'paper exercise' that restricts the quality of information generated from the process. ${ }^{4}$ Ocloo $e t a t^{\theta}$ in their recent narrative review 
of patient and public involvement (PPI) in healthcare call for 'models and frameworks that enable power and decision-making to be shared more equitably with patients and the public in designing, planning and co-producing healthcare'.

PPE is particularly important in the implementation of innovation and healthcare delivery improvements. ${ }^{10}$ Technology advancement such as new diagnostics play an important role in such innovation but development must be in keeping with patient's health needs and expectations. ${ }^{11}{ }^{12}$ More tests are making the translation from the laboratory to point-of-care test (POCT) devices, creating new diagnostic strategies that may disrupt traditional clinical pathways. It is rare for a patient to directly benefit from a diagnostic test in isolation; ${ }^{13}$ instead the impact comes from the decisions and interventions that are undertaken as a result of its introduction. Therefore, to allow informed input from public and patients into the research and development of novel diagnostic tests, the test process and any subsequent effects to downstream clinical pathways must be demonstrated.

Sequential simulation $(\mathrm{SqS})$ is a tool that demonstrates key elements in a patient's journey and can highlight the consequences of intervention at each step. SqS workshops can provide an innovative framework for PPE, especially when aligned to the four key assumptions of the Shared Immersion Model as described by Tang et $a:^{14}$

- Public engagement activities can be experiential, involving participation as well as acquisition of information

- The shared experience constitutes an event

- Being immersed in the shared experience is central, for researchers and publics alike. Critical evaluation of this process is equally valuable for both

- Simulation can provide immersive engagements between science and publics, especially with healthcare activity and research

Simulation has previously been used by our group as a tool for engagement with integrated care,${ }^{15}$ multidisciplinary teams and adolescents ${ }^{16}$ and for surgical devices. ${ }^{17}$ In the first two examples, SqS addressed clinical care pathway redesign; while in the latter, the focus was on the use of novel devices in healthcare environments. The current study combines these two approaches to facilitate PPE in the development of POCT diagnostics by exploring the stakeholder perspective on a new diagnostic test device and investigating its potential impact on the patient's journey.

The aim of this study is to introduce, through a case study, SqS as a methodology for active PPE in the development of novel POCTs and appraise the approach.

\section{CASE STUDY}

Oesophagogastric (OG) cancer represents the fourth and fifth most common types of cancer death. ${ }^{11}$ Each year in England, 12900 people are diagnosed with OG cancer and of these only $37.3 \%$ are considered curable at the time of diagnosis. ${ }^{11}{ }^{12}$ However, when diagnosed at its earliest stage, the 1-year survival is $75-87 \% .^{11}$ Public awareness of these cancer types is poor and the symptoms (dyspepsia (heartburn or indigestion), dysphagia (difficulty swallowing), unexplained weight loss, persistent vomiting, tiredness (anaemia) and upper abdominal pain) are common and often not associated with cancer. ${ }^{10}$ This leads to a delay in presentation to healthcare services and therefore treatment. In view of this, National Health Service (NHS) England recently undertook a 'Be Clear on Cancer' campaign for OG cancer targeting patients with heartburn and indigestion. ${ }^{18}$ Within our department, research is investigating the use of volatile organic compounds (VOCs) for early OG cancer diagnosis. Previous experimental work has demonstrated that exhaled breath analysis using mass spectrometry can distinguish oesophageal and gastric adenocarcinoma from non-cancer controls, ${ }^{19}$ demonstrating the potential for a VOC-based breath test to provide point-of-care risk stratification for patients with suspected OG cancer. This new approach aims to provide a readily available, non-invasive and cost-effective test to streamline patients with the early symptoms of cancer to further investigation with endoscopy. It is hoped that by increasing the number of patients diagnosed early, a higher proportion of these patients will have early stage disease and be able to enter a treatment pathway with curative intent. Much remains to be learnt about the social, cultural and practical implications of this new technology.

\section{METHODS}

Three 3-hour workshops were undertaken in central London. For workshop 1, we invited members of the Oesophageal Patients Association through their mailing list and social media, providing access to participants with prior experience of OG cancer and its diagnostic pathway, either personally or as a relative or carer. Workshops 2 and 3 were aimed at the general public without previous experience of OG cancer. Recruitment to workshop 2 was through an open invitation advertised in venues local to the workshop, and in workshop 3 a focus group recruitment firm (Focus4People, Herts, UK) was used.

The three workshops followed the same structure and comprised presentations, clinical pathway $\mathrm{SqS}$ and facilitated group discussions. Following a brief introduction to the day, a presentation covering the background to OG cancer including the current diagnostic strategy was given. This led into the first clinical pathway simulationa scenario that was designed to represent a relatively common patient journey. It was unscripted and followed a storyboard of a patient, played by a professional actor, noticing the symptoms of an oesophageal cancer and undergoing a typical diagnostic pathway (figure 1). The 

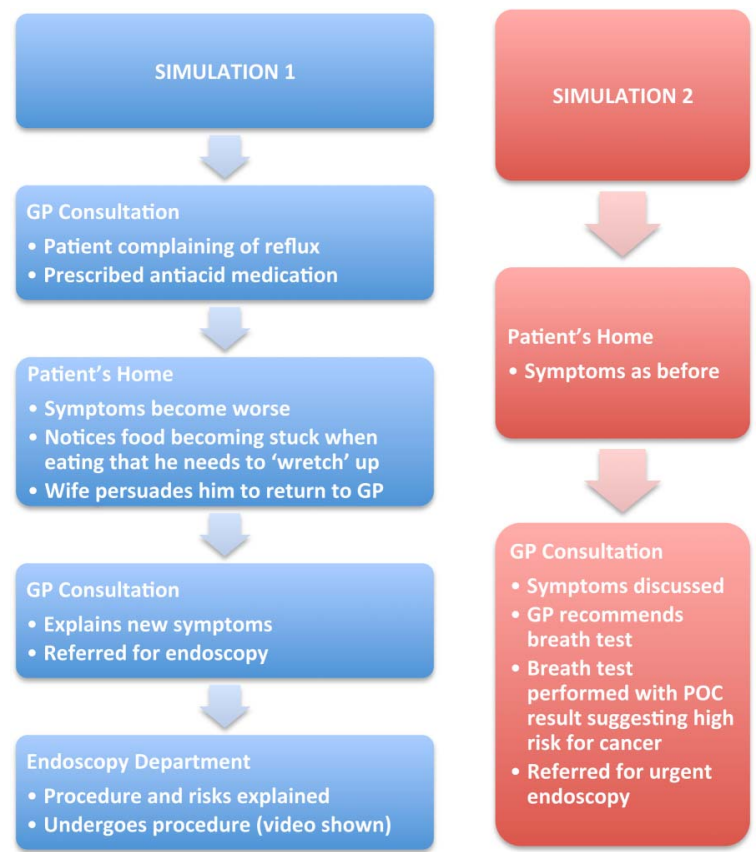

Figure 1 Storyboard of diagnostic pathway for oesophagogastric cancer used in sequential simulation scenarios. GP, general practitioner; POC, point-of-care.

storyboards were designed to represent a 'typical' uncomplicated diagnostic pathway for a patient with oesophageal cancer and created in consultation with specialist clinicians. To validate the storyboard participants with a personal experience of OG cancer, were asked to feedback on how the scenario related to their own experiences during the first workshop. Actors were used to ensure a personalised approach to the scenario, while maintaining confidentiality and ensuring consistency across workshops. Clinicians played their own healthcare roles in the simulation scenarios. The clinicians also respond well to actors who appear very realistic. The sets were created using distributed simulation ${ }^{20}$-versatile transportable screens and props including beds, desks and medical equipment (figure 2). The simulation scenario was followed by the workshop breaking down into small groups (5-10 people) for facilitated table discussions regarding the current diagnostic pathway. The groups were then brought back together and table discussions were summarised in a plenary session. After a coffee break, a member of the research team gave a presentation describing the current research achievements in lay terms and introduced the concept of the novel breath test, as well as our future ambitions. A second SqS followed the same patient through his journey, although this time we incorporated the breath test into the scenario to demonstrate the potential downstream consequences to the clinical pathway. Again, small table discussions followed.

Participants completed pre-event (see online supplementary appendix 1) and postevent (see online supplementary appendix 2) questionnaires that included a

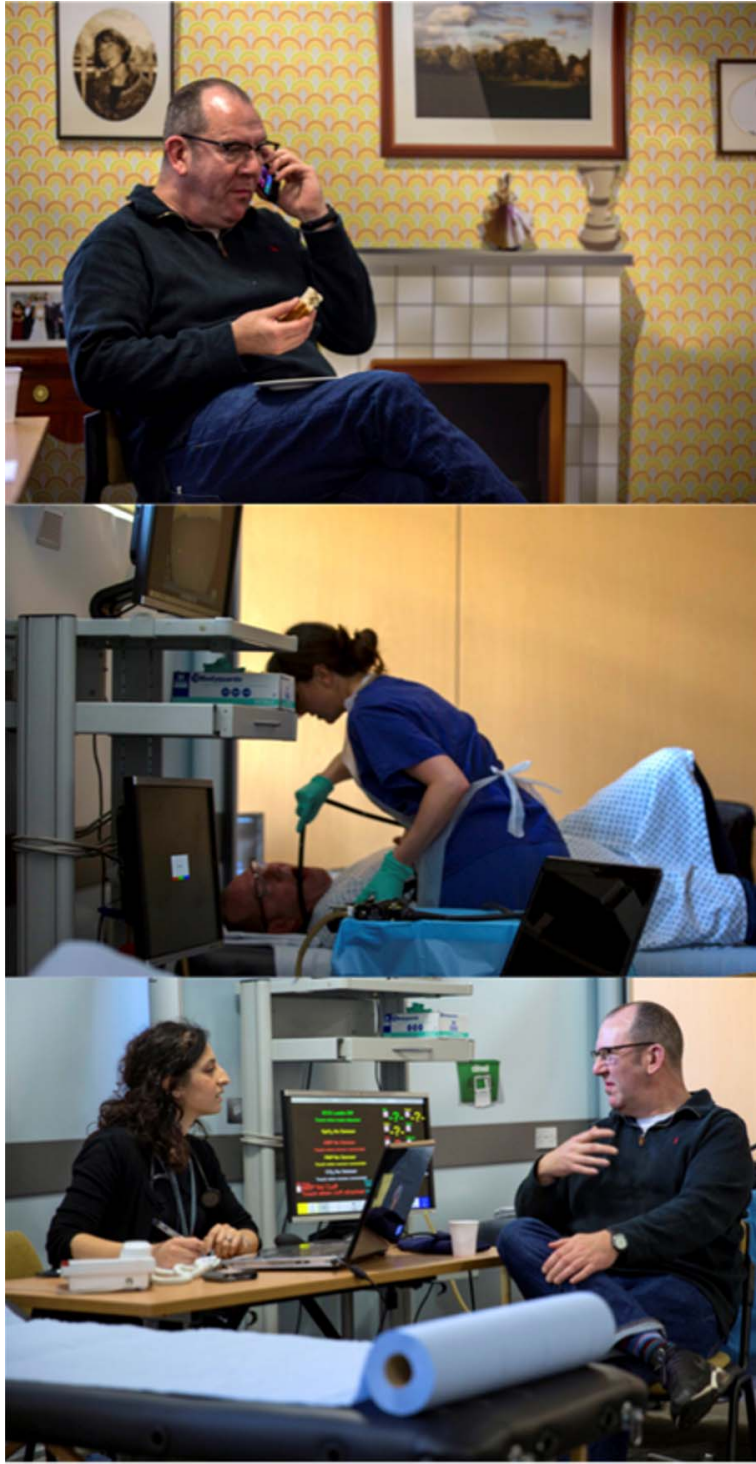

Figure 2 Sequential simulation (SqS) sets to illustrate (i) the patient's home (ii) an endoscopy unit and (iii) general practice consultation.

series of statements regarding attitudes and feedback related to the novel test device and also on the $\mathrm{SqS}$ workshop experience itself that participants were asked to rate on a 5-point Likert scale (strongly disagree to strongly agree). Participants were given copies of presentation slides and contact details if they would like to discuss anything from the workshop further.

The workshop discussions were filmed and recorded for subsequent analysis. Workshop recordings were transcribed and emergent thematic analysis undertaken independently by two members of the research team (JRH and SR), who were not directly involved in the test device research. Qualitative data were analysed with NVivo V.10.1.1 software (QSR International, Melbourne, Australia). Ethical approval for the workshops was provided by the Imperial College Joint Research Compliance Office (reference number ICREC_11_5_8) 
and informed consent was obtained from all workshop participants.

\section{RESULTS}

In total, 38 participants attended the 3 workshops, 26 $(68 \%)$ of whom had no previous experience personally or as a relative or carer of the OG cancer diagnostic pathway. A total of 12 participants were recruited through the Oesophageal Patients Association, of which $11(92 \%)$ attended; 14 participants replied to public poster and email campaign, of which $8(57 \%)$ attended; and 20 patients were recruited by the focus group recruitment company, of which $19(95 \%)$ attended. Two participants have subsequently volunteered to contribute further to our research activities and now have a role in PPI within the group.

\section{Thematic analysis of workshop audio recordings}

Two analyses were undertaken: (i) an analysis of participant attitudes towards the use of SqS for PPE in POCT diagnostics; and (ii) an analysis of patient feedback regarding the breath test device, diagnostic strategy and clinical pathway.

Three themes emerged regarding the use of SqS for public engagement in novel POCT diagnostic test: participatory engagement, simulation and empathetic engagement, and why participants attended. Theme summaries and quotations from transcripts are included in table 1.

In respect to the diagnosis of OG cancer and the proposed new breath test and diagnostic test strategy, five themes emerged from the workshop: awareness of OG cancer, barriers to testing and diagnosis, design of new test device, new clinical pathway and placement of test device. Theme summaries and participant quotations are presented in table 2 .

Table 3 demonstrates the "co-construction of knowledge' document. This collective output of shared experiences from researchers, patients and public summarises the key outputs raised from the workshops with proposed solutions to guide future research and implementation strategies.

\section{Participant feedback questionnaire}

All patients completed a questionnaire at the start and on completion of the workshop.

Feedback relating to the SqS workshop experience is shown in figure 3. All patients reported they felt able to contribute to the discussion (strongly agree $\mathrm{n}=31(82 \%)$; agree $\mathrm{n}=7(18 \%))$, that presentations were at a level they were able to understand (strongly agree $n=32(84 \%)$;

Table 1 Summary of themes relating to use of sequential simulation for public engagement in novel diagnostics

\begin{tabular}{|c|c|c|}
\hline $\begin{array}{l}\text { Key themes } \\
\text { identified }\end{array}$ & Summary & Quotations from transcript \\
\hline $\begin{array}{l}\text { Participatory } \\
\text { engagement }\end{array}$ & $\begin{array}{l}\text { All participants actively contributed to small group } \\
\text { discussions that often overran and continued into } \\
\text { breaks and over lunch. }\end{array}$ & $\begin{array}{l}\text { 'We were made comfortable to contribute'. W2 } \\
\text { 'Feeling that the patient perspective is important } \\
\text { in achieving better results'. W2 }\end{array}$ \\
\hline $\begin{array}{l}\text { Simulation and } \\
\text { empathetic } \\
\text { engagement }\end{array}$ & $\begin{array}{l}\text { Participants felt the simulations gave structure to } \\
\text { the events allowing them to focus on the } \\
\text { simulated patients' journey and understand the } \\
\text { consequences of the new diagnostic strategies. } \\
\text { Participants who had been through the diagnostic } \\
\text { pathway of OG cancer commented how similar it } \\
\text { was to their experiences although there were } \\
\text { some comments that the pathway was } \\
\text { oversimplified in some areas, for example, ease of } \\
\text { access to GP appointment and ease in which the } \\
\text { GP undertook the test within a consultation. }\end{array}$ & $\begin{array}{l}\text { 'The process I just watched echoed exactly what I } \\
\text { experienced when I was diagnosed'. W1 } \\
\text { 'The simulation of patient/GP scenarios to add } \\
\text { context'. W2 } \\
\text { 'It really stays in your mind, you know, you have a } \\
\text { picture'. W2 } \\
\text { 'It makes it more real'. W2 }\end{array}$ \\
\hline $\begin{array}{l}\text { Why participants } \\
\text { attended }\end{array}$ & $\begin{array}{l}\text { Attendance at the workshops was linked to the } \\
\text { method of recruitment. In the second workshop } \\
\text { that was recruited through advertisements in the } \\
\text { local areas (see methods), we explored the } \\
\text { motivation for attendance. This was broadly } \\
\text { divided into those with a community interest in } \\
\text { new diagnostic and medical developments and } \\
\text { those that had experience of cancer either } \\
\text { personally or as family or carers and wanted to } \\
\text { learn more and contribute to research in this area. }\end{array}$ & $\begin{array}{l}\text { 'I am interested in this way of, it's not only } \\
\text { researchers and doctors thinking about how to } \\
\text { develop it, but they are also listening carefully to } \\
\text { how people would feel with these new } \\
\text { developments'. W2 } \\
\text { 'This (workshop) interested me because as it just } \\
\text { so happens last year I had three gastroscopies } \\
\text { and I know what they feel like' W2 } \\
\text { 'I organize health workshops with community } \\
\text { development, with community organisations, we } \\
\text { work in the local community and we stress } \\
\text { prevention and that sort of thing'. W2 }\end{array}$ \\
\hline
\end{tabular}

W1, W2 or W3 denotes workshop where quotation was made.

GP, general practitioner; OG, oesophagogastric; W, workshop. 
Table 2 Summary of themes relating to current and novel diagnostic strategy for Oesophago-gastric cancer

\begin{tabular}{|c|c|}
\hline Key themes identified & Summary \\
\hline $\begin{array}{l}\text { Awareness of } \\
\text { oesophagogastric } \\
\text { cancer }\end{array}$ & $\begin{array}{l}\text { There was general agreement regarding the } \\
\text { lack of awareness of OG cancer, it's symptoms } \\
\text { and the poor outcome. There was a strong } \\
\text { feeling of a need for awareness campaigns } \\
\text { including celebrity endorsement and social } \\
\text { media. The role of the media in raising } \\
\text { awareness was discussed, and it was felt that } \\
\text { over the counter antacid medications should } \\
\text { contain warnings of the disease. }\end{array}$ \\
\hline
\end{tabular}

\section{Quotations from transcript}

'It needs to be more like colorectal cancer because people know if they have blood in their stools it's a worrying sign and straight away they go to their GP'. W1

'Media really important'! W2

'GPs see this condition rarely compared with other cancers'. W1

'If you are buying so and so amounts of Gaviscon this could be a sign (of cancer)... please check with your GP'. W3

'I didn't realise there was such a cancer at such a low survival rate'. W3

'Males more difficult to get to present and further awareness needed and less-invasive options'. W2

'How invasive the current test is could put a lot of people off'. W1 cultural reluctance to seeking medical advice particularly among men in at risk age groups, difficulty in accessing primary care services, delayed recognition of potential cancer and subsequent referral by GPs and hospital delays for investigation.

Design of new test device A potential urine test and breath test were acceptable, but overall most participants would prefer a breath test. There was a preference for a compact box like test similar to a police breathalyser (offers familiarity). Participants would rather see the test launched early and not delayed for attempts to improve accuracy. How the test provided results was a controversial topic with some participant preferring a quantitative result, for example, a risk percentage, some would prefer to have triage-like results, for example, high risk or low risk. A pure binary result, for example, red light/ green light was not popular as it was felt this would increase anxiety. What overrides these discussions was that the practitioner delivering the result should be appropriately trained to explain the meaning of the result and council regarding further management and privacy and support need to be provided. Written information was not felt to be sufficient and the inclusion of a nurse was highlighted as beneficial.

New clinical pathway proposed new pathway incorporating the potential breath test device. Participants felt that increasing access and convenience to diagnosis would encourage uptake, particularly in a non-invasive test device. There was some concern that a positive breath test would increase anxiety preceding endoscopy, but this was felt to be unavoidable and would always occur in cancer diagnostic pathways and may in fact increase the uptake of subsequent endoscopy. It was important that patients with a negative breath test know to return to their GP, if symptoms do not improve.

'It is important that people are trained to give out the result appropriately'. W1

'The simpler, the better'. W1

'Person giving results has to be able to quantify the risk (eg, percentage chance) of cancer when they give the test result- "high-risk" is too vague'. W1

'It is important that people are trained to give out the results appropriately'. W1

'I actually liked the box with a mouthpiece rather than a plastic bag'. W3

'I don't mind what kind of test as long as it's accurate'. W3

'Needs to be brought to market faster'

'Something before the invasive endoscopy test would encourage people more'. W1

'If there was a recurring acid problem and the breath test was negative I would probably want to go for an endoscopy'. W3

'The anxiety has to come at a point so maybe he just has to go through it earlier'. W1 
Table 2 Continued

\begin{tabular}{|c|c|c|}
\hline Key themes identified & Summary & Quotations from transcript \\
\hline $\begin{array}{l}\text { Placement of novel test } \\
\text { device }\end{array}$ & $\begin{array}{l}\text { Most participants felt the test should be placed } \\
\text { in either a GP surgery or a pharmacy. Other } \\
\text { proposed testing locations were: } \\
\text { Public places for 'drop-in' testing } \\
\text { Workplace testing } \\
\text { Health fairs } \\
\text { - Mobile testing to 'at-risk' populations } \\
\text { Booths in GP practices } \\
\text { Home testing }\end{array}$ & $\begin{array}{l}\text { 'If there is an easy test maybe it should be } \\
\text { available in a more accessible place than the } \\
\text { GP practice'. W1 } \\
\text { 'If the test is to be used in a pharmacy perhaps } \\
\text { just having high risk or low risk is sufficient, or } \\
\text { perhaps just that you need to have more tests or } \\
\text { you don't'. W1 } \\
\text { 'Are we going to have the approach like the } \\
\text { radiographers that do imaging and then say they } \\
\text { can't interpret the results and you will go to your } \\
\text { (doctor's) appointment'. W1 }\end{array}$ \\
\hline
\end{tabular}

W1, W2 or W3 denotes workshop where quotation was made.

GP, general practitioner; OG, oesophagogastric; W, workshop.

Table 3 Coconstruction of knowledge from researcher-participant shared experience

\begin{tabular}{ll}
\hline Issues raised & Solutions \\
\hline Poor awareness of OG cancer & Better media campaigns (a media campaign was initiated by NHS \\
& England during the course of this study), involvement of celebrities \\
and social media & Highlighting symptoms of OG cancer on antacid medication packets \\
& akin to health warnings on cigarette packaging \\
Invasiveness and risks of endoscopy deterring & Breath test could triage for endoscopy \\
patients presenting to medical services & Improve cost-effectiveness of patient journey \\
Breath test device design & Preference for compact (box like) simplistic breath test over urine \\
& Significant number of people not concerned about aesthetic \\
Positioning of test: pharmacy versus GP & appearance \\
& Preference for breath test over urine \\
& Preference for pharmacy for ease of access and speed in which test \\
& can be done \\
Concerns regarding meaning of test result and how it will be \\
-
\end{tabular}

GP, general practitioner; OG, oesophagogastric; NHS, National Health Service.

agree $\mathrm{n}=6(16 \%))$ and that the day was a useful experience (strongly agree $\mathrm{n}=31 \quad(82 \%) ; \mathrm{n}=7 \quad(18 \%))$. The majority of participants felt that diagnostic test devices will be better if patients are involved in their development (strongly agree $\mathrm{n}=26(68 \%)$; agree $\mathrm{n}=8 \quad(21 \%)$; neither agree or disagree $n=3(8 \%)$; disagree $n=1(3 \%))$.

Feedback relating to the breath test strategy is shown in figure 4. All patients liked the idea of a breath test for OG cancer (strongly agree $n=32(84 \%)$; agree $n=6(16 \%)$ ) and stated they would like a breath test before an endoscopy (strongly agree $n=36(95 \%)$; agree $n=2(5 \%))$. The majority of patients did not agree that the breath test would add to their anxiety (agree $\mathrm{n}=2 \quad(5 \%)$; neither agree or disagree $\mathrm{n}=7 \quad(18 \%)$; disagree $\mathrm{n}=10 \quad(26 \%)$; strongly disagree $n=16(42 \%) ; 3(8 \%)$ did not answer) and agreed that a 'low-risk' result from the breath test would provide reassurance without specialist referral (strongly agree $\mathrm{n}=7(18 \%)$; agree $\mathrm{n}=19(50 \%)$; neither agree or disagree $n=9(24 \%)$; disagree $n=3(8 \%))$.

\section{DISCUSSION}

SqS workshops provided an effective immersive environment for public and patient engagement around device development and future diagnostic strategy, creating a shared experience that was beneficial to participants 


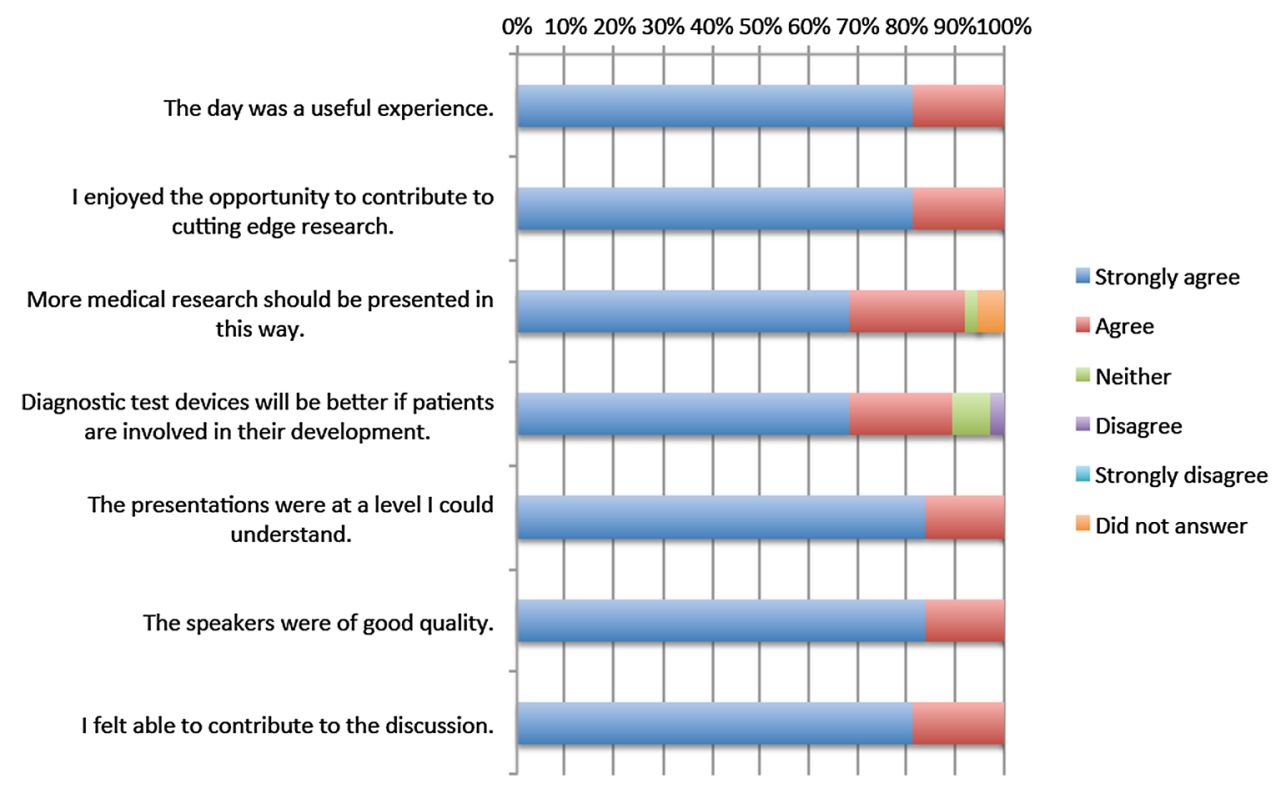

Figure 3 Participant questionnaire feedback relating to the sequential simulation (SqS) workshop experience.

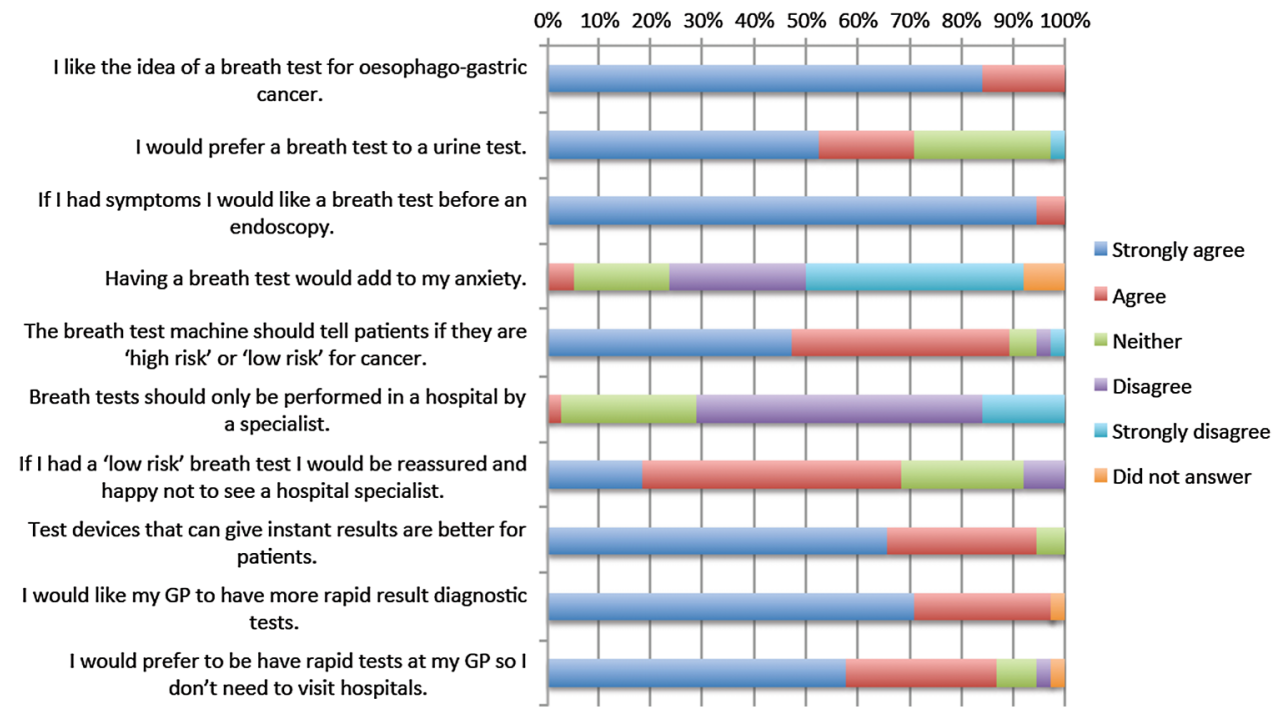

Figure 4 Participant questionnaire feedback relating to the novel breath testing strategy for the diagnosis of oesophagogastric cancer. GP, general practitioner.

and researchers. Rich data provide feedback on research activities to date and will help guide future research activities.

The outputs from the current workshops have already influenced future research plans regarding the VOCs breath test for OG cancer as follows: identifying a broader scope of where the test could be located, including general practice, pharmacies and workplaces; how the test result is presented to manage associated patient anxiety; and the migration from currently used breath bags to sensor technology. The workshops confirmed patient acceptability of the proposed breath testing strategy for cancer diagnosis and reinforced the lack of awareness that exists with regard to OG cancer and its symptoms. ${ }^{21}$ Furthermore, the workshop outputs have provided valuable information to other stakeholders concerned with POCT invention and adoption, including device designers, clinicians, commissioners and policymakers. As such, we believe results from PPE activities should be routinely published alongside clinical studies and cost-effectiveness arguments as part of a complete evidence package.

The largest challenge in running the workshops was recruitment. Three strategies were trialled, providing an opportunity to weigh the benefits and challenges of each and to recruit different participants with different backgrounds and motivations. The professional focus group recruitment firm was convenient and allowed the tailoring of the group to ensure a representative sample. However, this entailed greater cost as a recruitment 
commission was paid to the company. Recruitment to workshop 1 was also straightforward as replies to invitations sent to members of the Oesophageal Patients Association were forthcoming. Workshop 2 provided the greatest challenge in recruitment, requiring members of the research group to explore the locality of the workshop venue and placing posters in a variety of venues to encourage interest. Response rate was low, and there was a high rate of non-attendance on the day.

Other costs associated with running the workshops were venue hire including refreshments and lunch, professional actor fees, scenery and props for the simulation scenarios, and transport of equipment. PPI workshops do have an associated cost, for these three events we allocated a budget of $£ 12000$ (although this included the purchase of equipment that can be reused in subsequent events); it is therefore vital that the cost of PPE activities be incorporated into research budgets and funding applications.

This methodology does have limitations. Patient engagement necessitates voluntary participation, and convenience sampling was unavoidable. By varying the recruitment strategy, incentives for attendance and previous knowledge of OG cancer, we hope to have achieved an overall representative sample in the course of the three events, although sociocultural and clinical characteristics of participants were not recorded. Screening participants for their level of engagement using the Patient Health Engagement Scale ${ }^{22}$ or Patient Activation Measure $^{6}$ would have provided a useful measure to interpret the comments of participants. The transcripts from the events were analysed by two researchers who were not directly involved with the development of the breath test or VOC research, minimising bias in emergent theme analysis. Finally, the test device under evaluation is at a relatively early stage of development and therefore the scenarios were based on assumptions.

Patient engagement is vital at this early stage to guide device development, ensure the proposed clinical pathway is acceptable to patients and guide future research activities. To be effective, patient engagement must not be seen as an isolated event and needs to continue alongside evidence generation and device development to improve the acceptability of POCT.

The value of PPE is increasingly recognised in the development of medical devices and care pathways. Barello et $a l^{7}$ in their systematic review of eHealth for Patient Engagement, describe PPE as multidimensional with behavioural, cognitive and emotional components. The review concludes that the majority of approaches to PPE do not facilitate engagement at all three levels. However, SqS is able to provide a holistic and systematic approach that fulfils all three as described in the shared immersion model ${ }^{14}$ through the participant's role in the experience (emotional), evaluation (cognitive) and participation in the ongoing process of device development (behavioural). Furthermore, the nature of the shared event allows a degree of empathy between all stakeholders present, so that the outputs from the workshops can truly be underpinned in further research and device development.

In conclusion, $\mathrm{SqS}$ provides an effective methodology for active public and patient engagement and, to a limited degree, involvement in research towards the development of new POCT diagnostic devices and testing strategies. The outputs of these events provide rich data that can be of use to a wide range of stakeholders and could form a routine part of the evidence base, informing the adoption of new POCT devices.

Twitter Follow Jeremy Huddy at @JeremyHuddy

Acknowledgements The authors would like to thank Sheraz R Markar, Tom Wiggins, Stefan Antonowicz and Sophie Doran for presenting at the engagement workshops, as well as the Oesophageal Patient's Association for their assistance in recruiting participants for the first workshop.

Contributors JRH conceived of the study and participated in its design and coordination, data collection and analysis and drafted the manuscript. S-MW and SR participated in the study design, data collection and analysis, and helped draft the manuscript. TP helped with interpretation of data and drafting the manuscript. GBH conceived of the study, participated in the design and coordination of the study, and helped draft the manuscript. RK conceived of the study, participated in the design of the study and helped draft the manuscript. FB conceived of the study, participated in the design of the study and helped draft the manuscript. All authors read and approved the final manuscript.

Funding This research was supported by the National Institute for Health Research (NIHR) Diagnostic Evidence Co-operative London at Imperial College Healthcare National Health Service (NHS) Trust. The views expressed are those of the authors and not necessarily those of the NHS, the NIHR or the Department of Health.

Competing interests All authors have completed the International Committee of Medical Journal Editors (ICMJE) uniform disclosure form at http://www.icmje. org/coi_disclosure.pdf and declare: this research was supported and funded by the National Institute for Health Research (NIHR) Diagnostic Evidence Co-operative London at Imperial College Healthcare NHS Trust, JRH is funded by the National Institute for Health Research (NIHR) Diagnostic Evidence Co-operative, S-MW is funded by the Wellcome Trust, Health Education North West London, National Institute of Health Research, RK and FB are founding shareholders and FB is Executive Director (unpaid) of Convincis.

Ethics approval Imperial College Joint Research Compliance Office.

Provenance and peer review Not commissioned; externally peer reviewed.

Data sharing statement No additional data are available.

Open Access This is an Open Access article distributed in accordance with the terms of the Creative Commons Attribution (CC BY 4.0) license, which permits others to distribute, remix, adapt and build upon this work, for commercial use, provided the original work is properly cited. See: http:// creativecommons.org/licenses/by/4.0/

\section{REFERENCES}

1. INVOLVE supports public involvement in NHS, public health and social care research. http://www.invo.org.uk/ (accessed 19 Aug 2015).

2. Lijmer JG, Mol BW, Heisterkamp S. Empirical evidence of design-related bias in studies of diagnostic tests. JAMA 1999;282:1061.

3. Thompson J, Barber R, Ward PR, et al. Health researchers' attitudes towards public involvement in health research. Health Expect 2009;12:209-20.

4. Domecq JP, Prutsky G, Elraiyah T, et al. Patient engagement in research: a systematic review. BMC Health Serv Res 2014;14:89. 
5. Coulter A, Ellins J. Effectiveness of strategies for informing, educating, and involving patients. BMJ 2007;335:24-7.

6. Hibbard JH, Stockard J, Mahoney ER, et al. Development of the Patient Activation Measure (PAM): conceptualizing and measuring activation in patients and consumers. Health Serv Res 2004;39:1005-26.

7. Barello S, Triberti S, Graffigna G, et al. eHealth for patient engagement: a systematic review. Front Psychol 2015;6:2013.

8. Gruman J, Rovner MH, French ME, et al. From patient education to patient engagement: implications for the field of patient education. Patient Educ Couns 2010;78:350-6.

9. Ocloo J, Matthews R. From tokenism to empowerment: progressing patient and public involvement in healthcare improvement. BMJ Qual Saf 2016;25:626-32.

10. Zuckerman B, Margolis PA, Mate KS. Health services innovation: the time is now. JAMA 2013;309:1113-14.

11. Positive technology as a driver for health engagement.-PubMedNCBI. http://www.ncbi.nlm.nih.gov/pubmed/23792833 (accessed 6 Apr 2016).

12. Graffigna G, Barello S, Triberti S, et al. Enabling eHealth as a pathway for patient engagement: a toolkit for medical practice. Stud Health Technol Inform 2014;199:13-21.

13. Bossuyt PM, Irwig L, Craig J, et al. Comparative accuracy: assessing new tests against existing diagnostic pathways. BMJ 2006;332:1089-92.

14. Tang JJ, Maroothynaden J, Bello F, et al. Public engagement through shared immersion: participating in the processes of research. Sci Commun 2013;35:654-66.
15. Weldon SM, Ralhan S, Paice E, et al. Sequential Simulation (SqS) an innovative approach to educating GP receptionists about integrated care via a patient journey--a mixed methods approach. BMC Fam Pract 2015;16:109.

16. Powell $P$, Sorefan $Z$, Hamilton $S$, et al. Exploring the potential of sequential simulation. Clin Teach 2016;13:112-18.

17. Brunckhorst $O$, Shahid S, Aydin A, et al. The relationship between technical and nontechnical skills within a simulationbased ureteroscopy training environment. J Surg Educ 2015;72:1039-44.

18. Be Clear on Cancer Oesophago-gastric cancers. http://www.nhs.uk/ be-clear-on-cancer/oesophagogastric-cancer/ (accessed 19 Aug 2015).

19. Kumar S, Huang J, Abbassi-Ghadi N, et al. Selected ion flow tube mass spectrometry analysis of exhaled breath for volatile organic compound profiling of esophago-gastric cancer. Anal Chem 2013;85:6121-8.

20. Kneebone R, Arora S, King D, et al. Distributed simulation--accessible immersive training. Med Teach 2010;32:65-70.

21. Tentzeris V, Lake B, Cherian $\mathrm{T}$, et al. Poor awareness of symptoms of oesophageal cancer. Interact Cardiovasc Thorac Surg 2011;12:32-4.

22. Graffigna G, Barello S, Bonanomi A, et al. Measuring patient engagement: development and psychometric properties of the Patient Health Engagement (PHE) Scale. Front Psychol 2015;6:274. 